Axe thématique : 1

\title{
Une éducation physique tournée vers l’activité physique, le bien-être et la santé en Fédération Wallonie-Bruxelles
}

Jérémy Bonni ${ }^{1} \&$ Marc Cloes $^{2}$

1. Haute École de la Province de Liège, Section AESI éducation physique (Liège, Belgique)

Université de Liège, Département des Sciences de la motricité (Liège, Belgique)

Résumé : À l'heure où les comportements sédentaires et l'inactivité physique ne cessent de croître dans la plupart des pays du monde et où les recommandations en matière d'activité physique ne sont que trop rarement atteintes (OMS, 2020; Wijtzes et al., 2016), la Fédération Wallonie-Bruxelles (FWB - Belgique) procède actuellement - à l'instar d'autres pays ayant déjà opéré ce changement depuis plusieurs années (exemples : Canada, Écosse) à une réforme de l'éducation physique, en passant d'une éducation physique dite citoyenne, à une éducation physique et à la santé. Dans ce contexte, le Ministère de l'Enseignement en FWB a mandaté un groupe d'experts et de chercheurs pour identifier, concevoir et partager des dispositifs et des outils visant à promouvoir l'activité physique, le bien-être et la santé en contexte scolaire. Sur base d'une démarche d'ingénierie didactique collaborative (Goigoux, 2012 ; Sénéchal, 2016), des pratiques dites « exemplatives » ont pu être identifiées, conçues, expérimentées et enfin, partagées sur la plateforme éducative " e-classe ", proposée par la FWB à toute sa communauté éducative. Cette publication tend finalement à questionner le(s) rôle(s) et la place du professeur d'éducation physique et à la santé au sein d'un établissement scolaire, dans un monde en pleine mutation et qui doit faire face à de nouveaux défis.

\section{Mots clés :}

Éducation physique ; Activité physique ; Bien-être ; Santé ; Curriculum.

\section{Introduction}

À l'heure actuelle, dans un monde en pleine évolution dans tous les domaines, nous remarquons une augmentation de l'inactivité physique et des comportements sédentaires partout dans le monde, renforçant inévitablement le risque de maladies non transmissibles, telles que les maladies cardio-vasculaires, le cancer ou encore le diabète ${ }^{1}$ (OMS, 2018).

En Belgique, alors que I'Organisation Mondiale de la Santé (OMS) recommande pour les enfants et adolescents âgés entre 5 et 17 ans - de pratiquer 60 minutes d'activité physique quotidienne d'intensité modérée à soutenue afin de préserver sa santé et contribuer à prévenir ces maladies (OMS, 2020), le « bulletin 2016 de la pratique d'activité physique chez les enfants et les adolescents belges " souligne qu'uniquement $7 \%$ des enfants âgés entre 6 et 9 ans et $2 \%$ des adolescents entre 10 et 17 ans rencontrent ces recommandations (Wijtzes et al., 2016).

\footnotetext{
${ }^{1}$ La sédentarité est l'un des 10 facteurs majeurs de risque de mortalité dans le monde (OMS, 2018).
} 
De plus, bien que l'école soit justement identifiée comme un lieu privilégié pour mettre en œuvre des interventions visant à augmenter la pratique d'activités physiques chez les enfants et les adolescents (McMullen et al., 2015), l'enseignement de l'éducation physique semble décliner dans le monde (UNESCO, 2017) alors qu'elle est considérée comme l'un des moyens les plus efficaces et accessibles pour promouvoir l'activité physique (Payne \& Morrow, 2009).

Face à ce constat alarmant, $56 \%$ des États Membres de l'OMS se sont ainsi dotés de politiques visant à lutter contre la sédentarité (OMS, 2018). En Fédération WallonieBruxelles (FWB), c'est par l'entremise d'une réforme majeure de l'enseignement ${ }^{2}$ visant à renforcer la qualité (l'efficacité et l'équité) de l'enseignement pour tous les élèves - dont le travail a débuté en 2015 - que s'inscrit la réforme de l'éducation physique actuelle. Dans un monde où notre santé est négligée (UNESCO, 2017), il s'avère en outre nécessaire $d^{\prime}$ 'intervenir auprès des élèves pour les aider à adopter des comportements favorables à leur bien-être et à leur santé (European Week of Sport EAC - Sport, 2017³). C'est pourquoi, l'implémentation d'actions en éducation à la santé sera en outre officialisée et renforcée dans les curriculums scolaires en FWB ${ }^{4}$.

Dans ce contexte, le Ministère de l'Enseignement a mandaté, depuis janvier 2017, un groupe d'experts et de chercheurs, le Consortium « Activités Physiques, Bien-Être et Santé » $\left(\mathrm{CAPBES}^{5}\right.$ ), issus des douze établissements d'enseignement supérieur (Universités et Hautes Écoles) impliqués dans la formation des futurs professeurs d'éducation physique, au bienêtre et à la santé ${ }^{6}$ en FWB, afin d'identifier, concevoir et partager des outils et dispositifs respectant à la fois les convictions et les axes du PEE, tout en promouvant l'activité physique, le bien-être et la santé chez les élèves.

L'identification de pratiques dites "exemplatives " qui ont fait leurs preuves et que les acteurs de terrain pourront s'approprier en fonction de leur contexte d'enseignement, de leurs caractéristiques respectives et de celles de leurs élèves mais également la conception et l'expérimentation de nouvelles pratiques - par rapport au contexte spécifique de la FWB - et leur partage, s'avèrent être trois objectifs primordiaux pour tendre vers une éducation physique (et à la santé) de qualité.

Parallèlement à ces trois principaux objectifs, deux questions de recherche soustendent le travail mené par ce groupe de recherche : (1) Comment influencer positivement l'activité physique, le bien-être et la santé des enfants/adolescents en contexte scolaire et dans leur vie quotidienne (actuelle et future) dans l'optique d'un ancrage sociétal (Cloes, 2017) ? ; (2) Dans quelle mesure le(s) rôle(s) et la place du professeur d'éducation physique (et à la santé) devront-ils évoluer pour s'ajuster aux modifications du curriculum ?

\footnotetext{
${ }^{2}$ Le Pacte pour un Enseignement d'Excellence (PEE) s'articule autour de quatre convictions fortes et de cinq axes stratégiques (voir l'avis $n^{\circ} 3$ du Groupe central, publié le 7 mars 2017 et disponible via le lien suivant : http://www.enseignement.be/index.php?page=28280).

${ }^{3}$ Déclaration de Tartu.

${ }^{4}$ Depuis septembre 2020, dans l'enseignement maternel et, à partir de septembre 2022, en première et deuxième année de l'enseignement primaire.

${ }^{5} \mathrm{Ou}$ "Consortium 7" du Pacte pour un Enseignement d'Excellence.

${ }^{6}$ Jusqu'à la fin du tronc commun mis en place jusqu'à la $3^{\mathrm{e}}$ secondaire (15 ans).
} 


\section{Méthodologie}

Une démarche d'ingénierie didactique collaborative (Goigoux, 2012 ; Sénéchal, 2016) a été préconisée afin d'atteindre ces trois principaux objectifs. La spécificité de cette démarche, se voulant représentative de la Fédération Wallonie-Bruxelles, tient dans la collaboration entre les formateurs-chercheurs et les enseignants de terrain pour la conception, l'expérimentation et la validation des pratiques et des outils développés.

L'identification de ces dispositifs et outils didactiques visant à promouvoir l'activité physique, le bien-être et la santé a été effectuée selon différentes méthodes. D'une part, une revue de la littérature portant sur l'inclusion de l'éducation à la santé dans les programmes d'éducation physique a été entreprise, à partir de ce qui est actuellement connu et validé scientifiquement. D'autre part, des dispositifs et outils basés sur des projets développés en FWB, correspondant aux priorités du PEE mais sans être nécessairement validés scientifiquement, ont été identifiés.

L'identification et l'analyse de ces dispositifs et outils se sont en outre basées sur le croisement de deux modèles. L'un portant sur les six piliers de l'activité physique en contexte scolaire (Syners et al., 2014) et l'autre sur les 28 objets d'enseignement et d'apprentissage en éducation à la santé (Turcotte et al., 2011).

Le modèle des six piliers de l'activité physique (Snyers et al., 2014) questionne le rôle du professeur d'éducation physique et à la santé et met en évidence six domaines $d^{\prime}$ intervention visant à influencer la pratique d'activité physique des élèves: le projet d'établissement qui constitue un pilier plus transversal puisqu'il devrait (si possible) reprendre des dispositifs d'enseignement/apprentissage mis en place au sein des autres piliers, en vue de les rendre pérennes ; le cours d'éducation physique (de qualité) ; l'activité physique et sportive extracurriculaire; les modes de déplacements actifs; l'aménagement de l'environnement scolaire; et l'activité physique en classe.

La classification des objets d'enseignement et d'apprentissage en éducation à la santé (Turcotte et al., 2011) repose sur l'analyse de contenus proposés dans le cadre de neuf programmes scolaires canadiens. Elle repose sur les quatre facteurs clés du développement individuel des enfants et des adolescents en matière de réussite éducative, de santé et de bien-être : l'estime de soi ; la compétence sociale; les habitudes de vie; ainsi que les comportements sains et sécuritaires (Turcotte et al., 2011).

En croisant ces deux modèles, le CAPBES s'est doté d'un outil permettant de classer les dispositifs didactiques mis en place en milieu scolaire dans la perspective de promouvoir la diversité des interventions (figure 1).

\section{Résultats}

75 fiches décrivant des dispositifs et des outils visant à promouvoir l'activité physique, le bien-être et la santé en contexte scolaire, considérées comme pertinentes par les membres du CAPBES et validées par les membres du Groupe central du Pacte pour un Enseignement d'Excellence, ont été répertoriées. La figure 2 illustre une partie du contenu de ces ressources. Ces dernières sont mises à la disposition des personnels de 


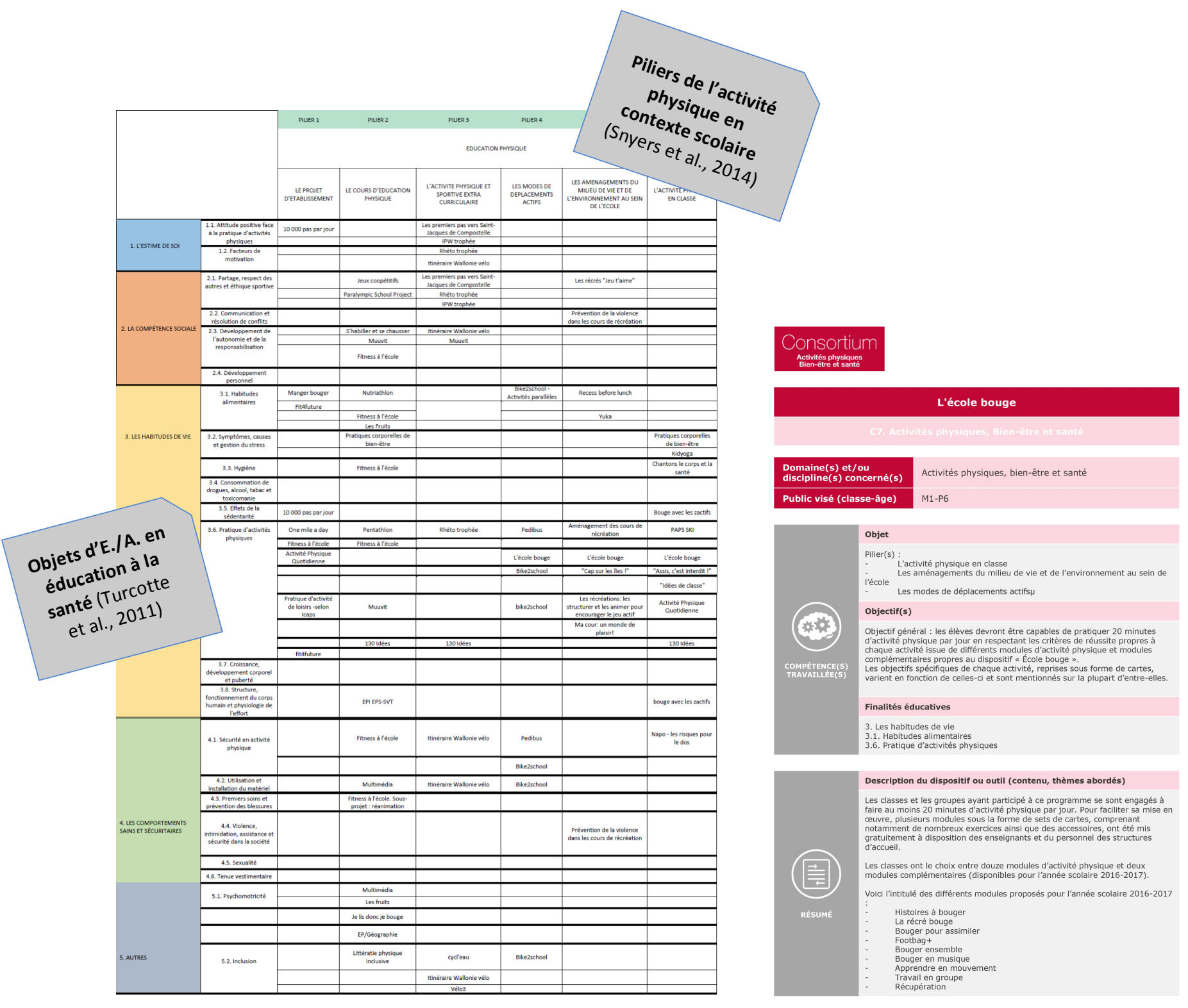

Figure 1 : Modèle bidimensionnel de classification des outils et dispositifs identifiés par le CAPBES

Figure 2 : Partie de fiche descriptive d'un dispositif identifié par le CAPBES

l'enseignement sur une plateforme éducative, intitulée " e-classe », proposée en ligne par la FWB à toute sa communauté éducative ${ }^{7}$. Parallèlement à l'identification de ces dispositifs et outils didactiques, six projets originaux ont été conçus et expérimentés dans le contexte spécifique propre à la $\mathrm{FWB}^{8}$.

\footnotetext{
${ }^{7}$ Plusieurs informations sont répertoriées dans ces fiches, telles que : les fondements pédagogiques qui soustendent le dispositif mis en place, les outils pédagogiques utilisés, les pratiques diagnostiques, de différenciation, voire d'individualisation prévues pour gérer l'hétérogénéité des élèves, la place du langage dans l'appropriation de l'apprentissage, la place accordée aux outils numériques et les résultats d'une éventuelle validation scientifique du dispositif (impact sur les élèves, degré de confiance des enseignants, etc.). ${ }^{8}$ Cinq de ces six projets ont d'ailleurs fait l'objet de communications orales/affichées lors de la SEPAPS 2020, dont voici les intitulés : (1) "Les aménagements de la cour et ses effets sur les élèves : I'indice de fidélité du dispositif en question"; (2) "Analyse d'un dispositif d'auto-évaluation de la condition physique à l'aide d'une application web"; (3) "Activité physique, connaissances en santé et habitudes alimentaires d'élèves belges: effets de la méthodologie Oblomov "; (4) "Mise en place de "Pauses d'Activités Physiques Scolaires 》 (PAPS) dans une commune rurale"; (5) "Mise en place d'un dispositif pédagogique visant l'amélioration du temps d'activité des enfants dans la cour de récréation ". Pour les consulter, il vous suffit de vous rendre dans l'espace dédié à la $11^{\mathrm{e}}$ Biennale de l'ARIS : https://events.uliege.be/sepaps2020/ressources-disponibles/.
} 


\section{Discussion et conclusion}

Au vu de ces travaux, la capacité d'un enseignant à influencer positivement l'activité physique, le bien-être et la santé de ses élèves - en contexte scolaire et pour leur vie quotidienne (actuelle et future) (Cloes, 2017) - pourrait ainsi dépendre des domaines d'intervention dans lesquels il sera en mesure d'agir (Snyers et al., 2014), mais également des objets d'enseignement et d'apprentissage visés et développés (Turcotte et al., 2011). Par ailleurs, les recherches effectuées dans le cadre de cette réforme de l'éducation physique questionnent naturellement le(s) rôle(s) et la place du professeur d'éducation physique et à la santé au sein d'un établissement scolaire. Dans quelle mesure un enseignant en éducation physique (et à la santé) devrait-il prendre part aux différents projets visant à promouvoir l'activité physique, le bien-être et la santé chez ses élèves ?

Dans un premier temps, il s'avérera probablement nécessaire de se concentrer sur son cours, en s'attelant à développer chez ses élèves les compétences reprises dans le(s) référentiel(s) officiels de la FWB, tout en veillant à respecter les principes d'un cours d'éducation physique de qualité. À cet égard, I'utilisation du modèle PAMIA (Cloes, 2019) pourrait s'avérer particulièrement pertinent puisqu'il identifie cinq composantes essentielles pour tendre vers un tel cours (Plaisir; Autonomie; Mouvement; Interactions; Apprentissage).

Dans un second temps, l'enseignant motivé et formé dans ce sens pourrait prendre part à l'organisation d'activités physiques et sportives extracurriculaires, stimuler et renforcer les modes de déplacement actifs, initier des changements dans son environnement scolaire (cours de récréation, couloirs, escaliers, classe) et encourager les collaborations et les activités interdisciplinaires en classe; en gardant les objets d'enseignement et d'apprentissage à la santé en toile de fond de toute action (Hills et al., 2015 ; Snyers et al., 2014 ; Turcotte et al., 2011).

Cependant, l'impact des enseignants en éducation physique et à la santé ne peut être dissocié des objectifs fixés dans le plan de pilotage de leur établissement scolaire et d'une intervention concertée de tous les membres d'une communauté éducative. En effet, seules des approches multisectorielles pourront réellement mener à des changements dans le mode de vie des enfants et adolescents qui deviendront ensuite adultes (Cale \& Harris, 2006 ; Cloes, 2017). L'arrimage et la complémentarité des interventions visant à promouvoir l'activité physique, le bien-être et la santé feront en effet la différence (Beddoes \& Castelli, 2017). Le professeur d'éducation physique et à la santé devrait dès lors être à l'initiative de telles actions, pour ensuite pouvoir les coordonner en accompagnant les autres acteurs éducatifs dans la création d'un environnement scolaire " actif "; en devenant ainsi, au sens où l'entendent Tappe et Burgeson (2004), la pierre angulaire de la promotion d'un mode de vie sain et actif.

À cet égard et sur base du modèle socio-écologique de l'intervention en éducation physique (ICAPS, 2017), les dispositifs et outils identifiés par les membres du CAPBES pourront s'avérer particulièrement utiles aux praticiens, sous réserve de certaines adaptations à réaliser de leur part, en fonction du contexte spécifique à leur école et leur(s) 
classe(s), de leurs propres caractéristiques et de celles de leurs étudiants. La plateforme éducative " e-classe 9 " les regroupe et les met à disposition, librement et gratuitement, à toute la communauté éducative de la FWB.

\section{Références}

Beddoes, Z., \& Castelli, D. M. (2017). Comprehensive school physical activity programs in middle schools. Journal of Physical Education, Recreation \& Dance, 88(6), 26-32.

Cale, L., \& Harris, J. (2006). School-based physical activity interventions: effectiveness, trends, issues, implications and recommendations for practice. Sport, Education and Society, 11(4), 401-420.

Cloes, M. (2017). Preparing physically educated citizens in physical education: Expectations and practices. Retos: nuevas tendencias en educación física, deporte y recreación, (31), 245-251.

Cloes, M. (2019). A four level model aiming to guide PE teachers to become key actors in changing youth' lifestyle. 8e Congresso Sociedade Cientifica de Pedagogia do Desporto. Coimbra (Portugal).

European Week of Sport EAC-Sport (2017). Declaration of Tartu Promoting healthy lifestyles among children and young people, notably by supporting schools and sport clubs in creating and developing partnerships. Retrieved from https://ec.europa.eu/sport/sites/sport/files/ewos-tartu-call_en.pdf

Goigoux, R. (2012). Didactique du français et analyse du travail enseignant. À quelles conditions la didactique ne deviendra-t-elle pas un luxe inutile ? In M.-L. Élalouf, A. Robert, A. Belhadjin et M.-F. Bishop (dir.), Les didactiques en question(s). État des lieux et perspectives pour la recherche et la formation (pp. 3342). Bruxelles : De Boeck.

Hills, A. P., Dengel, D. R., \& Lubans, D. R. (2015). Supporting public health priorities: recommendations for physical education and physical activity promotion in schools. Progress in cardiovascular diseases, 57(4), 368-374.

ICAPS (2017). Argumenter l'intérêt de développer un projet de promotion de l'activité physique auprès des jeunes et de limiter la sédentarité. Retrieved from http://moocaps.santepubliquefrance.fr/mooc/

McMullen, J., Ni Chroinin, D., Tammelin, T., Pogorzelska, M., \& Van Der Mars, H. (2015). International approaches to whole-of-school physical activity promotion. Quest, 67(4), 384-399.

OMS (2020). Lignes directrices de l'OMS sur l'activité physique et la sédentarité. Genève : OMS. Retrieved from https://www.who.int/fr/publications-detail/9789240014886

Payne, V. G., \& Morrow, J. R. (2009). School physical education as a viable change agent to increase youth physical activity. The President's Council on Physical Fitness and Sports Research Digest, 10(2), 1-8.

Sénéchal, K. (2016). Expérimentation et validation de séquences didactiques produites selon une ingénierie didactique collaborative : L'enseignement de la discussion et de l'exposé critique au secondaire. Thèse de doctorat, Université Laval, Québec.

Snyers, J., Halkin, A-S., Lejacques, T., Schmit, J., Williot, J., \& Cloes, M. (2014). Multidimensional analysis of the importance given to physical activity promotion in secondary schools of French-speaking Belgium. The Global Journal of Health and Physical Education Pedagogy, 3(3), 212-227.

Tappe, M.K. \& Burgeson, C.R. (2004). Physical Education: A Cornerstone for physically active lifestyles. Journal of Teaching in Physical Education, 23(4), 281-299.

Turcotte, S., Desbiens, J-F., Trudel, C., Demers, J., \& Roy, M. (2011). L'inclusion de l'éducation à la santé dans les programmes canadiens d'éducation physique. Revue phéneEPS, 3(1), 1-21.

UNESCO (2017). Plan d'action de Kazan - MINEPS VI. SHS/2017/PI/H/14 REV (p.7). Retrieved from http://unesdoc.unesco.org/images/0025/002527/252725f.pdf

Wijtzes, A.I., Verloigne, M., Mouton, A., Cloes, M., De Ridder, K.A.A., Cardon, G., \& Seghers, J. (2016). Results from the 2016 Active Healthy Kids Belgium Report Card on Physical Activity for Children and Youth. Journal of Physical Activity and Health, 13(Suppl 2), S95 -S103. doi: 10.1123/jpah.2016-0306.

\footnotetext{
${ }^{9}$ Lien direct : https://www.e-classe.be/.
} 